\title{
Transperineal versus transrectal prostate biopsy for predicting the final laterality of prostate cancer: are they reliable enough to select patients for focal therapy? Results from a multicenter international study
}

R. Miano, C. De Nunzio, F.J. Kim, B. Rocco, P. Gontero, C. Vicentini, S. Micali, M. Oderda, S. Masciovecchio, A.D. Asimakopoulos

Division of Urology, Department of Experimental Medicine and Surgery, Fondazione Policlinico Tor Vergata (RM) and Policlinico Casilino (ADA), University of Tor Vergata; Department of Urology, Sant'Andrea Hospital, University of La Sapienza (CDN), Rome, Italy; Division of Urology, Department of Surgery, Denver Health Medical Centre (FJK), Denver, CO, USA; Clinica Urologica I, Università degli Studi di Milano, Fondazione IRCCS Ca' Granda - Ospedale Maggiore Policlinico (BR), Milan; Department of Urology-1, A.O.U. San Giovanni Battista, University of Turin (PG, MO), Turin; Division of Urology, Ospedale Civile G.Mazzini, University of L'Aquila (CV, SM), Teramo and Department of Urology, University of Modena and Reggio Emilia, Policlinico di Modena (SM), Modena, Italy

\section{ABSTRACT}

Objectives: To compare the concordance of prostate cancer (PCa) laterality between the extended transperineal (TP) or transrectal (TR) prostate biopsy (BP) and radical prostatectomy (RP) specimens. To identify predictors of laterality agreement between BP and RP.

Materials and Methods: Data from 533 consecutive patients with PCa (278 TP and 255 TR-diagnosed) treated with RP were analyzed. A 12-core technique was used for both TP and TR biopsies. Additional cores were obtained when necessary.

Results: Overall, the percentage of agreement of PCa laterality between BP and RP was $60 \%(K=0.27, p<0.001)$. However, the RP confirmation of unilaterality at BP was obtained in just 33\% of the cases. Considering the concordance on bilaterality as the "target" of our analysis, the sensitivity and specificity were 54.3\% and 98.2\%, respectively, with TP and 47.5\% and 92.5\%, respectively with TR. Focusing on patients with unilaterality at biopsy, none of the evaluated preoperative variables (biopsy technique, age, total positive biopsy cores, PSA, prostate volume, Gleason score on biopsy) were able to predict RP bilaterality in the multivariate analyses.

Conclusions: Most of the patients with unilateral involvement at BP harbored bilateral PCa after RP. TR and TP biopsy showed no difference in their capacity to predict the concordance of tumor laterality at RP. None of the preoperative evaluated variables can predict the tumor laterality at RP. Using BP unilaterality to include patients in focal therapy (FT) protocols may hinder the oncologic efficacy of FT.

\section{ARTICLE INFO}

Key words:

Prostatic Neoplasms; Biopsy; therapy [Subheading]

Int Braz J Urol. 2014; 40: 16-22

Submitted for publication:

March 29, 2013

Accepted after revision: November 12, 2013 


\section{INTRODUCTION}

Recent pathologic studies from contemporary radical prostatectomy $(\mathrm{RP})$ series have cited frequencies of unifocal prostate cancer $(\mathrm{PCa})$ ranging from $10 \%$ to $44 \%$ and unilateral PCa ranging from 10\% to $40 \%$ (1). In this context, focal therapy (both focal ablation and hemiablation) has progressively gained interest as a new method to control clinically localized PCa and has been defined as 'a type of treatment that aims to eradicate known cancer within the prostate and, at the same time, spare uninvolved prostatic tissue, with the aim of preserving genitourinary function' (2). Consequently, the accurate preoperative assessment of PCa is crucial and the exact spatial location of the tumor is essential to deliver treatment.

Transrectal ultrasound (TRUS)-guided biopsy of the prostate, performed through the transrectal (TR) or the transperineal (TP) approach is the current standard for the diagnosis of PCa and to define the localization of the disease. Several studies have investigated the degree of concordance in tumor laterality between prostate biopsy (BP) and RP (3-11) and its implication for focal therapy $(3,4,6,8-11)$. However, in the majority of these studies the BP was performed through the transrectal approach; no data have been published on the transperineal approach, while the use of the perineal template has been recently described, although the procedure is more invasive and has health-care resource implications $(2,12,13)$. Multiparametric MRI is receiving a great interest in the last years having the performance characteristics required to localize significant areas of prostate cancer, but the routine use of this technique can not be proposed for clinical (expertise of the radiologist) and health-care (cost and diffusion of MRI) implications (12).

The aim of the current study was to compare the concordance in the tumor laterality between the contemporary extended pattern prostate biopsy performed via a transperineal (TP) or transrectal (TR) approach and RP specimens in patients with PCa. Secondarily, we sought to identify predictors of laterality agreement between the BP and RP.

\section{MATERIALS AND METHODS}

Data from 533 consecutive patients with PCa diagnosed after an extended biopsy (278 via
TP approach and 255 via TR approach) who underwent RP were retrospectively analyzed. Seven different urology departments participated in the study. Three of these departments performed the TR biopsies (Denver, CO, USA; Rome, Italy; Teramo, Italy), and the others performed the TP biopsies (Rome; Milan; Turin; Modena, Italy).

TRUS guidance was used in all cases. The biopsy procedure was performed with an 18G biopsy needle in both TR and TP cases under local anesthesia. A 12-core technique was used in the TP biopsies ( 6 cores per lobe), covering the peripheral zone of both lobes from the lateral to the paramedian area and from the base to the apex. A 12-core technique was also followed for the TR biopsies, using the same scheme (two cores from the lateral peripheral zone, two from the paramedian-lateral zone and two from the paramedian apical and basal zone per lobe).

For prostate volumes $>50 \mathrm{~mL}$, two more peripheral cores (one from the lateral zone and one from the paramedian-lateral) were added. Additional cores from the anterior part or from the transition zone of the prostate were taken, depending on the clinical and ultrasound characteristics.

$\mathrm{RP}$ was performed according to the surgical principles of each center. Each center had a dedicated uropathologist examining the specimens (both of $\mathrm{BP}$ and RP). At all the participating centers, RP specimens were en-bloc formalin fixed and inked to delineate the surgical margins; a step-sectioned transversally at $2-4 \mathrm{~mm}$ intervals technique was used to examine the specimen; an apical shaved-section, 2-4mm thick, was truncated perpendicular to the prostatic urethra and sub-sequentially sectioned as slices parallel to the prostatic urethra. Bladder neck was examined sampling portions of tissue at the junction of the prostatic capsule and bladder neck or by sampling the most proximal portion of the submitted specimen corresponding to the anatomical bladder neck. The 2002-TNM classification was used.

The pathologic evaluation of the BP cores reported the ratio of total positive cores/total cores, laterality and the Gleason sum and score. The pathologic evaluation of the RP specimens reported the laterality, Gleason sum and score, pathologic stage and margin status. 


\section{Statistical analysis}

The comparison of the patients' baseline characteristics between the TR and TP groups was performed in agreement with the statistical distribution of the variables (chi squared for nominal variables, Mann-Whitney for ordinal variables, t-test after log-transformation for interval scales such as PSA and prostate volume).

The concordance between the tumor laterality as assessed by BP and by RP was measured using the typical diagnostic indexes: sensitivity, specificity and accuracy. The chi-squared test was used to compare the diagnostic performance of the two groups (TR and TP).

A multiple logistic-regression with bilaterality at RP as the binary dependent variable and the biopsy approach (TP vs. TR), age, total positive specimens, PSA, Gleason score on biopsy and prostate volume as the independent categorical or continuous covariates was performed on cases with unilaterality at biopsy. The interactions between group-total positive specimens, group-prostate volume and group-pathological stage were also analyzed.

\section{RESULTS}

The demographic and baseline characteristics are summarized in Table-1. As shown, the two groups of patients did not differ in terms of age, PSA levels, total biopsy cores, biopsy total Gleason score or prostate volume.

Overall, the percentage of correct classifications of the tumor laterality by biopsy with respect to prostatectomy was 60\%. The Kappa measure of agreement was equal to 0.27 . This value was statistically significant $(\mathrm{p}<0.001$, thus rejecting the null hypothesis of lack of agreement). Specifically, we found that the overall prostatectomy confirmation of unilaterality at biopsy occurred only in 103 out of 311 cases (33\%). Thus, the total agreement of 60\% and the significant Kappa measurement were primarily due to the cases classified as bilateral by biopsy and confirmed to be bilateral after RP (217/222 = 97.7\%). Considering the concordance of bilaterality (between the BP and RP) as the "target" of our analysis, the overall biopsy sensitivity was low (51.1\%), while the specificity was quite high (95.4\%). When the BP suggested bilaterality, this was almost always true, while the BP indication of unilaterality was confirmed at RP in only 1 out of 3 cases.

We observed similar patterns when comparing TP and TR. The percentage of correct concordance in laterality was 62.9\% (Карpa $=0.31, \mathrm{p}<$ $0.001)$ with TP and 56.9\% (Kappa $=0.23, \mathrm{p}<0.001)$ with TR. The sensitivity and specificity with respect to bilaterality were $54.3 \%$ and $98.2 \%$, respectively, with TP and 47.5\% and 92.5\%, respectively, with TR (Table-2). Even if TP showed slightly higher diagnostic performance, TP was not significantly better than TR in terms of sensitivity ( $p=0.165)$, specificity $(p=0.157)$ and accuracy $(p=0.152)$.

The occurrence of a concordance between biopsy and prostatectomy was not dependent on the number of total BP cores (OR $=0.99, \mathrm{p}=0.717$ ).

Focusing our analysis on cases classified as unilateral at biopsy, we sought to identify potential predictors of misclassification. Overall, none of the evaluated preoperative variables (biopsy technique, age, total positive biopsy cores, PSA, prostate volume, Gleason score on biopsy) was able to predict the bilaterality of the final pathology on the multivariate analyses (Table-3). Moreover, age, total positive biopsy cores, PSA, prostate volume and Gleason score on biopsy were not independent predictors of bilateral PCa within the TP and TR groups when analyzed separately.

\section{DISCUSSION}

In recent years, due to the stage migration of PCa, there has been an increased interest in alternative strategies that offer the possibility of delaying, obviating or minimizing the impact of radical treatments (such as RP or radiotherapy), while maintaining the same oncologic long-term results. One such strategy is active surveillance with selective delayed intervention. Despite the advantages (avoiding overtreatment and complications in patients with low-risk PCa) and disadvantages (risk of progression and psychological and healthcare burdens) that have recently been addressed, definitive results from ongoing randomized clinical trials are required to assess whether active surveillance should be routinely implemented in clinical practice (14). 
Table 1 - Baseline characteristics of the two groups (TP and TR) of patients.

\begin{tabular}{|c|c|c|c|c|c|c|c|c|c|}
\hline & \multicolumn{4}{|c|}{$\operatorname{TP}(n=278)$} & \multicolumn{4}{|c|}{$\operatorname{TR}(\mathrm{n}=255)$} & \multirow[b]{2}{*}{ t-test(df), p-value } \\
\hline & Mean & SD & $\begin{array}{l}95 \% \\
\mathrm{Cl} \text { inf }\end{array}$ & $\begin{array}{l}95 \% \mathrm{Cl} \\
\text { sup }\end{array}$ & Mean & SD & $\begin{array}{c}95 \% \mathrm{Cl} \\
\text { inf }\end{array}$ & $\begin{array}{l}95 \% \mathrm{Cl} \\
\text { sup }\end{array}$ & \\
\hline Age & 64.6 & 5.8 & 63.9 & 65.3 & 64.0 & 6.2 & 63.2 & 64.7 & $1.192, p=0.234$ \\
\hline $\begin{array}{l}\text { Total PSA } \\
(\mathrm{ng} / \mathrm{ml})^{\star}\end{array}$ & 8.6 & 5.3 & 8.0 & 9.3 & 8.6 & 4.1 & 8.1 & 9.1 & $0.184, p=0.854$ \\
\hline $\begin{array}{l}\text { Total biopsy } \\
\text { cores }\end{array}$ & 12.6 & 5.3 & 11.9 & 13.2 & 12.2 & 3.5 & 11.7 & 12.9 & $1.524, p=0.342$ \\
\hline $\begin{array}{l}\text { Prostate } \\
\text { volume } \\
(\mathrm{mL})^{\star}\end{array}$ & 38.9 & 29.7 & 35.3 & 42.4 & 42.3 & 16.7 & 40.3 & 44.5 & $1.738, p=0.084$ \\
\hline $\begin{array}{l}\text { Biopsy Total } \\
\text { Gleason } \\
\text { score }\end{array}$ & 6.6 & 0.9 & 6.5 & 6.7 & 6.4 & 0.7 & 6.3 & 6.5 & $1.826, p=0.068$ \\
\hline $\begin{array}{l}\text { RP Total } \\
\text { Gleason } \\
\text { score }\end{array}$ & 6.9 & 1.0 & 6.8 & 7.1 & 6.7 & 0.9 & 6.6 & 6.8 & $2.590, p=0.010$ \\
\hline pT stage & & & & & & & & & \\
\hline $\begin{array}{l}\mathrm{T}_{2}: \mathrm{n}=329 \\
(61.7 \%)\end{array}$ & & \multicolumn{3}{|c|}{$183(65.9 \%)$} & & \multicolumn{2}{|c|}{$146(57.3 \%)$} & & \\
\hline $\begin{array}{l}\mathrm{T}_{3}: \mathrm{n}=193 \\
(36.2 \%)\end{array}$ & & \multicolumn{2}{|c|}{$93(33.3 \%)$} & & & \multicolumn{2}{|c|}{$100(39.2 \%)$} & & Chi-Square $(2)=7.82, p=0.020$ \\
\hline $\begin{array}{l}\mathrm{T}_{4}: \mathrm{n}=11 \\
(2.1 \%)\end{array}$ & & \multicolumn{2}{|c|}{$2(0.7 \%)$} & & & \multicolumn{2}{|c|}{$9(3.5 \%)$} & & \\
\hline
\end{tabular}

Table 2 - Agreement between biopsy and prostatectomy in terms of tumor laterality.

\begin{tabular}{lccc}
\hline Pathologic evaluation & \multicolumn{2}{c}{ Prostatectomy $(n=533)$} \\
\hline PCa laterality & $\begin{array}{c}\text { Unilateral }(n) \\
(\% \text { concordance })\end{array}$ & $\begin{array}{c}\text { Bilateral }(n) \\
(\% \text { concordance })\end{array}$ \\
\hline TP $(n=278)$ & Unilateral $(n=156)$ & $54(34.6)$ & $102(65.4)$ \\
TR $(n=255)$ & Bilateral $(n=122)$ & $1(0.8)$ & $121(99.2)$ \\
& Unilateral $(n=155)$ & $49(31.6)$ & $106(68.4)$ \\
& Bilateral $(n=100)$ & $4(4)$ & $96(96)$ \\
\hline
\end{tabular}


Table 3 - Multivariate analysis.

\begin{tabular}{lcccc}
\hline & Odds Ratio & \multicolumn{2}{c}{$95 \%$ C.I.for OR } & p-value \\
\hline & & Lower & Upper & \\
\hline TR vs. TP & 1.33 & 0.59 & 3.04 & 0.492 \\
Age (years) & 1.02 & 0.96 & 1.08 & 0.517 \\
Total positive biopsy cores (n) & 1.05 & 0.96 & 1.16 & 0.273 \\
Total PSA (log) & 1.01 & 0.58 & 1.78 & 0.964 \\
Prostate volume (log) & 1.04 & 0.47 & 2.29 & 0.926 \\
Biopsy Gleason score 7 vs. $<=6$ & 0.63 & 0.30 & 1.32 & 0.219 \\
Biopsy Gleason score $>$ 7 vs. $<=6$ & 0.82 & 0.19 & 3.61 & 0.797 \\
\end{tabular}

Focal treatment may be an acceptable alternative for low-risk PCa, representing a compromise between the ambiguity of surveillance and the potential reduction of the quality of life of a radical treatment; aiming to destroy only the areas of PCa, focal therapy (FT) could deliver cancer control while simultaneously avoiding damage to the surrounding structures (15). This may reduce incontinence, impotence, and rectal toxicity.

Whether current hypotheses regarding the efficacy of FT suggest that it may be effective so long as it treats the "index lesion", even if other lesions remain untreated in the gland, the optimal patient selection criteria for FT are not known and are therefore not standardized. Ongoing FT trials for PCa include the laterality, Gleason score and tumor volume as criteria for patient selection $(1,12,16)$. The role of ultrasound and multiparametric MRI to define the exact disease localization is still debatable (12).

Currently, TRUS-guided transrectal BP remains the widespread approach to evaluate patient suitability for FT protocols in terms of tumor topography, volume and grading. The accuracy of transrectal BP for predicting PCa laterality has been widely evaluated $(3-9,11)$. All these studies but one (4) agree that almost two thirds of the patients with unilateral PCa at BP harbor bilateral PCa at RP and consequently $\mathrm{BP}$ is considered as inadequate for the purposes of candidate selection for FT.

Only Polaschik et al. (4) suggested that BP unilaterality may be used to select men with low- to low-moderate-risk PCa for hemiablation FT. In their study, although less than one third of the patients had confirmed unilateral disease at the final pathological evaluation, the strongest predictor of pathologic unilaterality was prostate biopsy unilaterality (odds ratio $[\mathrm{OR}]=3.88 ; 95 \%$ confidence interval [CI], 2.14-7.05; $\mathrm{P}<0.0005$ ).

Furthermore, in these studies, none of the biopsy or clinical features, including the PSA, PSA ratio, clinical stage, gland volume, number of positive biopsy cores, high grade prostatic intraepithelial neoplasm, Gleason score, perineural invasion, percentage of positive cores) could clearly and independently predict the presence of unilateral PCa in univariate and multivariate logistic regression models.

Increasing the number of BP cores is not traduced in improving the accuracy of TRUS biopsy in predicting laterality. Abdollah et al (8) conducted a retrospective study on 203 patients who underwent an initial TRUS prostate saturation biopsy (24 cores) followed by RP. They concluded that initial saturation BP is not sufficiently accurate as a method of predicting tumor laterality in RP specimens and that the use of saturation biopsy to guide hemi-ablation therapy of PCa may lead to mistreatment in a considerable proportion of patients. Again, none of the routinely available clinical and pathological characteristics appeared to improve the ability of unilateral $\mathrm{PCa}$ on biopsy to predict unilateral $\mathrm{PCa}$ in the RP specimen. The same conclusions were also reached by Falzarano et al (9) in a study based on 72 patients 
who underwent RP after saturation biopsy. Only 4 of 39 patients (10\%) with a unilateral positive TRUS saturation biopsy had unilateral cancer at the final pathological evaluation. Our study confirmed the poor role of extended TRUS biopsy to predict PCa laterality at final pathology, with a concordance rate of 31.6\% for unilateral disease between BP and RP. The overall statistical significance of the Kappa measurement of agreement that we obtained was rather reached due to the large sample size because a value of 0.27 conventionally indicates low agreement (17).

Is the extended TP biopsy more accurate for mapping $\mathrm{PCa}$ ?

To our knowledge, only an abstract by Hoshi et al (18) prospectively evaluated 147 consecutive men who subsequently underwent RP. The PPV of transperineal saturation BP (the probability that the tumor is unilateral on the final pathological exam when it was unilateral on preoperative biopsy) was only 30\% and consequently this approach was not sufficiently accurate to detect unilateral PCa and it should not be used to decide which patients should be offered FT. However, a full-text manuscript did not follow this abstract.

Template transperineal prostate mapping biopsies might provide more exact information about the spatial tumor distribution of PCa and might accurately identify unilateral cancer for the purpose of FT $(13,19,20)$. When used with a $5 \mathrm{~mm}$ sampling frame, this approach can rule in and rule out PCa foci of $0.5 \mathrm{cc}$ and $0.2 \mathrm{cc}$ volumes, with $90 \%$ certainty (19). There exists unanimous agreement that the current gold standard for characterizing men who are considering FT is transperineal BP using a template-guided approach, although it is not yet widely available and imposes a high burden on healthcare services because of its requirement for anesthesia, pathology processing and slower reporting time (2). Moreover, correlation with whole-mount pathological specimens is necessary to fully analyze the accuracy of this technique, but to date, no study is available.

Our study did not demonstrate any significant advantage of the transperineal approach over the TR in the prediction of the laterality concordance of cancer. Even if TP showed slightly higher diagnostic performance, TP was not significantly better than TR in terms of sensitivity, specificity and ac- curacy. When unilateral cancer was diagnosed after transperineal biopsy, the unilaterality was confirmed in only $34.6 \%$ of the patients, suggesting that, as with the transrectal approach, two thirds of the patients had bilateral cancer that was only detected at final pathology.

Finally, none of the evaluated preoperative variables (biopsy technique, age, total positive biopsy cores, PSA, prostate volume and Gleason score on biopsy) were able to predict bilaterality at the final pathology in the multivariate analyses. Moreover, age, total positive biopsy cores, PSA, prostate volume and Gleason score on biopsy were not independent predictors of bilateral PCa within the TP and TR groups when analyzed separately.

The present study is the first to report the accuracy of TP extended biopsy in predicting the laterality of $\mathrm{PCa}$, and it is the first to compare TR versus TP in the same setting, with a standardized biopsy scheme. The absence of data on the clinical stage and the pathologic tumor volume as well as the absence of comparison on the complication rates and costs of the two bioptic approaches are the main shortcomings of our study.

\section{CONCLUSIONS}

TRUS-guided biopsy is not accurate for predicting PCa laterality when compared to the RP pathology result. The majority of patients with unilateral involvement at biopsy harbor bilateral PCa; the transperineal approach does not show any advantages over the transrectal in the capacity to predict the concordance of tumor laterality at radical prostatectomy. None of the preoperative evaluated variables can predict the final laterality. Thus, using unilaterality as a parameter for treating patients with FT may lead to mistreatment. Future research should aim to improve imaging techniques (such as multiparametric MRI) to obtain an accurate map of the prostate and understanding the biological implications of the different PCa foci for focusing treatment on the significant ("index") lesions.

\section{ACKNOWLEDGEMENTS}

International Translational Research in Uro-Sciences Team (ITRUST) 


\section{CONFLICT OF INTEREST}

None declared.

\section{REFERENCES}

1. Karavitakis M, Winkler M, Abel P, Livni N, Beckley I, Ahmed $\mathrm{HU}$ : Histological characteristics of the index lesion in wholemount radical prostatectomy specimens: implications for focaltherapy. Prostate Cancer Prostatic Dis. 2011; 14: 46-52.

2. de la Rosette J, Ahmed H, Barentsz J, Johansen TB, Brausi $M$, Emberton $M$, et al.: Focal therapy in prostate cancer-report from a consensus panel. J Endourol. 2010; 24: $775-80$.

3. Tareen B, Godoy G, Sankin A, Temkin S, Lepor H, Taneja SS: Can contemporary transrectal prostate biopsy accurately select candidates for hemi-ablative focal therapy of prostatecancer? BJU Int. 2009; 104: 195-9.

4. Polascik TJ, Mayes JM, Schroeck FR, Sun L, Madden JF, Moul JW, et al.: Patient selection for hemiablative focal therapy of prostate cancer: variables predictive of tumor unilaterality based uponradical prostatectomy. Cancer. 2009; 115: 2104-10.

5. Isbarn H, Karakiewicz PI, Vogel S, Jeldres C, Lughezzani G, Briganti A, et al.: Unilateral prostate cancer cannot be accurately predicted in low-risk patients. Int J Radiat Oncol Biol Phys. 2010 ; 77: 784-7.

6. Quann P, Jarrard DF, Huang W: Current prostate biopsy protocols cannot reliably identify patients for focal therapy: correlation of low-risk prostate canceron biopsy with radical prostatectomy findings. Int J Clin Exp Pathol. 2010 ; 3: 401-7.

7. Mayes JM, Mouraviev V, Sun L, Tsivian M, Madden JF, Polascik TJ: Can the conventional sextant prostate biopsy accurately predict unilateral prostate cancer in low-risk, localized, prostatecancer? Urol Oncol. 2011; 29: 166-70.

8. Abdollah F, Scattoni V, Raber M, Roscigno M, Briganti A, et al.: The role of transrectal saturation biopsy in tumour localization: pathological correlation after retropubic radicalprostatectomy and implication for focal ablative therapy. BJU Int. 2011; 108: 366-71.

9. Falzarano SM, Zhou M, Hernandez AV, Moussa AS, Jones JS, Magi-Galluzzi C: Can saturation biopsy predict prostate cancer localization in radical prostatectomy specimens: a correlative study andimplications for focal therapy. Urology. 2010; 76: 682-7.

10. Sinnott M, Falzarano SM, Hernandez AV, Jones JS, Klein EA, Zhou M, et al.: Discrepancy in prostate cancer localization between biopsy and prostatectomy specimens in patients with unilateral positivebiopsy: implications for focal therapy. Prostate. 2012; 72: 1179-86.
11. Gallina A, Maccagnano C, Suardi N, Capitanio U, Abdollah $\mathrm{F}$, Raber M, et al.: Unilateral positive biopsies in low risk prostate cancer patients diagnosed with extended transrectal ultrasound-guidedbiopsy schemes do not predict unilateral prostate cancer at radical prostatectomy. BJU Int. 2012; 110: E64-8.

12. Valerio $\mathrm{M}$, Ahmed $\mathrm{HU}$, Emberton M, Lawrentschuk $\mathrm{N}$, Lazzeri M, Montironi R, et al.: The Role of Focal Therapy in the Management of Localised Prostate Cancer: A Systematic Review. Eur Urol. 2013 6. pii: S0302-2838(13) 00557-5.

13. Crawford ED, Rove KO, Barqawi AB, Maroni PD, Werahera PN, Baer CA, et al.: Clinical-pathologic correlation between transperineal mapping biopsies of the prostate and threedimensionalreconstruction of prostatectomy specimens. Prostate. 2013; 73: 778-87.

14. Lawrentschuk N, Klotz L: Active surveillance for low-risk prostate cancer: an update. Nat Rev Urol. 2011; 8: 312-20.

15. Lecornet E, Ahmed HU, Moore CM, Emberton M: Conceptual basis for focal therapy in prostate cancer. J Endourol. 2010; 24: 811-8.

16. Taneja SS, Mason M: Candidate selection for prostate cancer focal therapy. J Endourol. 2010; 24: 835-41.

17. Hale CA, Fleiss JL: Interval estimation under two study designs for kappa with binary classifications. Biometrics. 1993; 49: 523-34.

18. Hoshi S, Yamamuro T, Ogata Y, Numahata K, Sugano 0. Transperineal saturation biopsy did not identify unilateral prostate cancer potentially amenable to focal treatment. $J$ Urol 2010; 183 (4): e55 \#Abstract 135.

19. Crawford ED, Wilson SS, Torkko KC, Hirano D, Stewart JS, Brammell $\mathrm{C}$, et al.: Clinical staging of prostate cancer: a computer-simulated study of transperineal prostate biopsy. BJU Int. 2005; 96: 999-1004.

20. Barzell WE, Melamed MR: Appropriate patient selection in the focal treatment of prostate cancer: the role of transperineal 3-dimensional pathologic mapping of the prostate--a 4-year experience. Urology. 2007; 70(6 Suppl): 27-35. 